\title{
0590. Impact of arterial tone changes on dynamic arterial elastance and the arterial pressure response to fluid administration
}

\author{
Ml Monge García ${ }^{1,2 *}$, M Gracia Romero², P Guijo González², A Gil Cano², J Mesquida ${ }^{2}$ R Andrew ${ }^{1}$, RM Grounds ${ }^{1}$, \\ M Cecconi ${ }^{1}$
}

From ESICM LIVES 2014

Barcelona, Spain. 27 September - 1 October 2014

\section{Introduction}

Dynamic arterial elastance $\left(\mathrm{Ea}_{\mathrm{dyn}}\right)$, the relationship between pulse pressure variation (PPV) and stroke volume variation (SVV), has been suggested as a functional assessment of arterial load for predicting the arterial pressure response after volume expansion $(\mathrm{VE})^{1}$. Although changes in $\mathrm{Ea}_{\mathrm{dyn}}$ have been related with variations in arterial load ${ }^{2}$, the effect of acute arterial tone changes on $\mathrm{Ea}_{\mathrm{dyn}}$ and the impact on its performance for predicting the arterial pressure response after VE has not yet been determined.

\section{Objective}

To evaluate the effect of acute arterial tone changes on $\mathrm{Ea}_{\mathrm{dyn}}$ and the influence on its performance for predicting arterial changes after fluid administration.

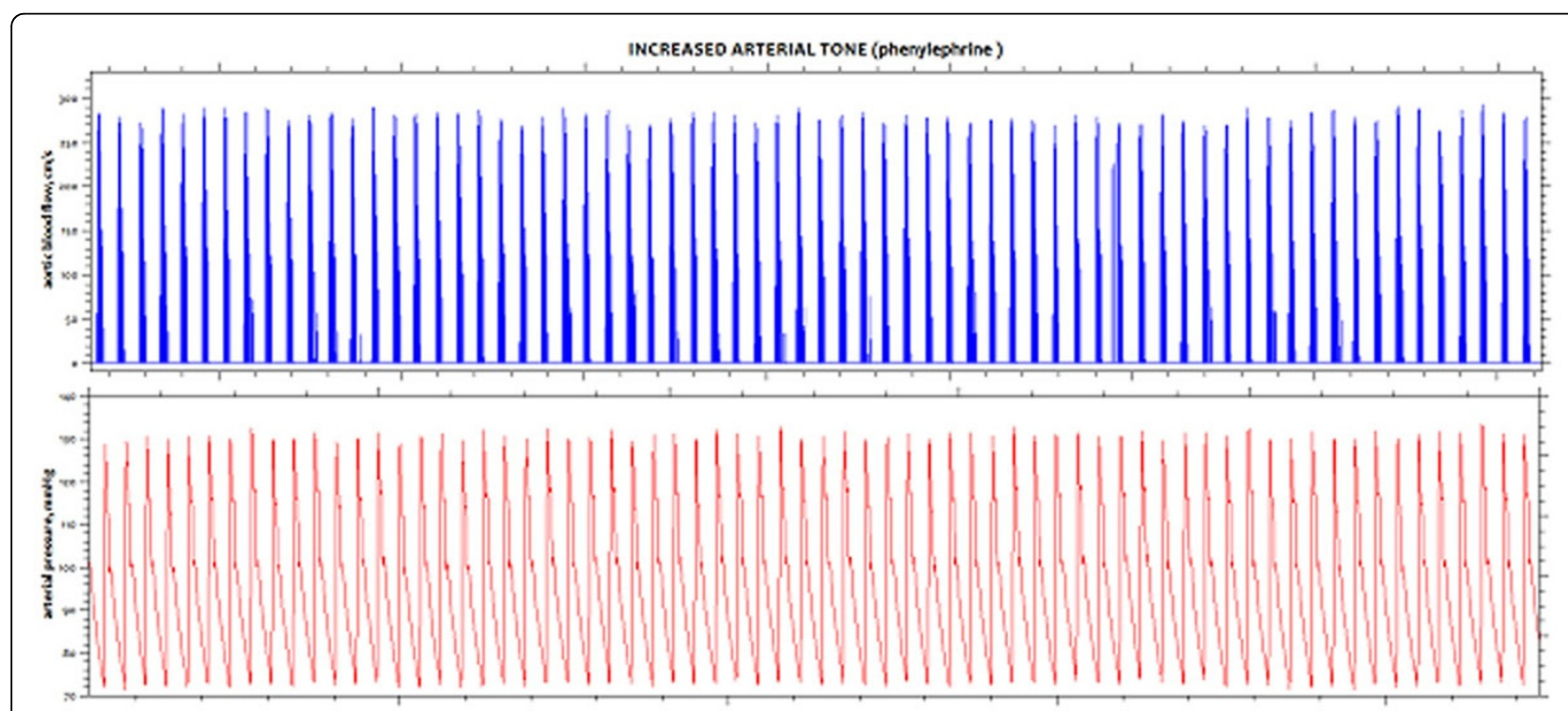

Figure $1 \mathrm{SW}$ and PPV example on HighMAP group.

'St. George's Healthcare NHS Trust and St George's University of London,

Department of Intensive Care Medicine, London, UK

Full list of author information is available at the end of the article

(C) 2014 García et al; licensee Springer. This is an Open Access article distributed under the terms of the Creative Commons Attribution License (http://creativecommons.org/licenses/by/2.0), which permits unrestricted use, distribution, and reproduction in any medium, provided the original work is properly cited. 


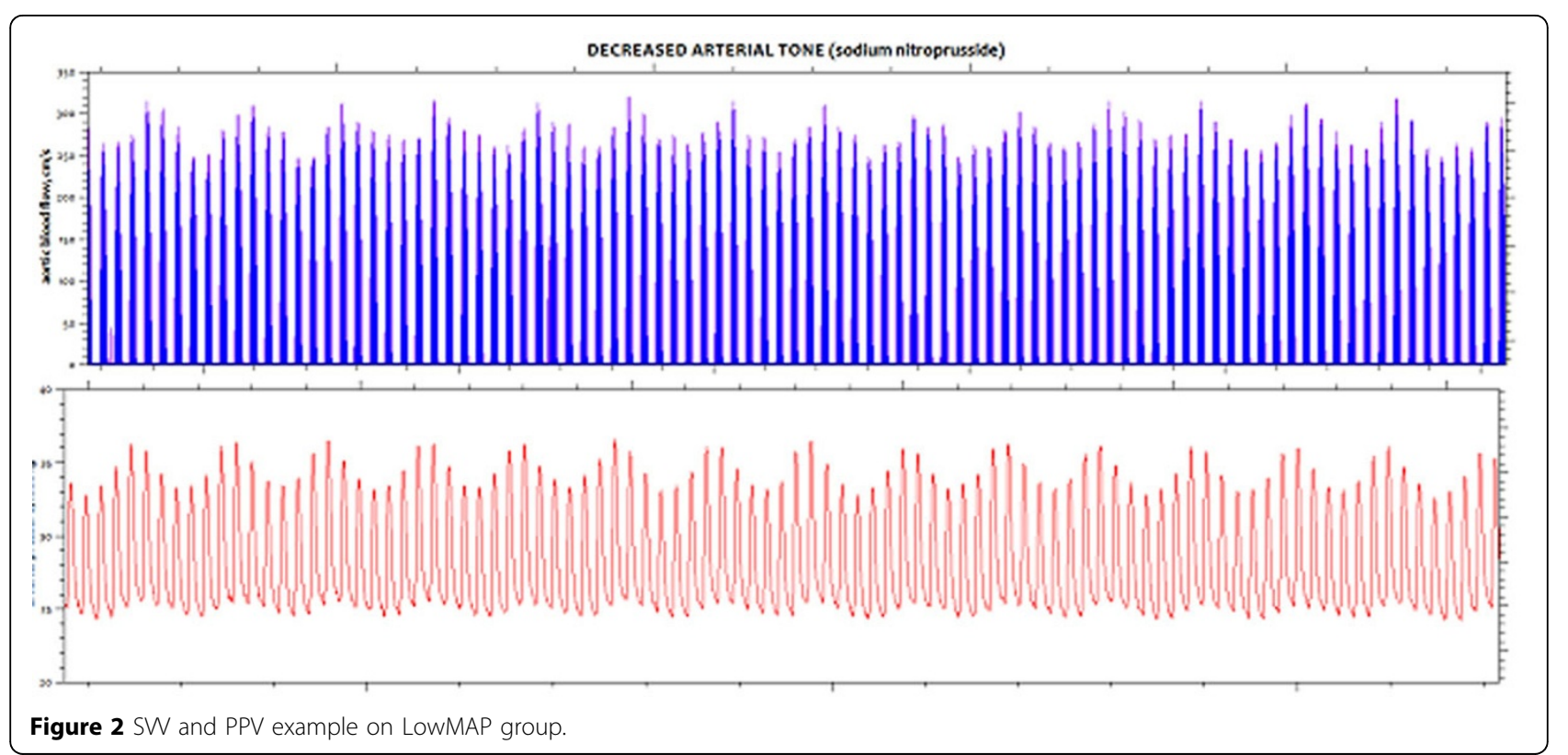

Table 1 Comparison of arterial load parameters during different experimental conditions in HighMAP abnd LowMAP groups ( $\mathrm{n}=6$ on both experimental arms).

\begin{tabular}{|c|c|c|c|c|}
\hline & Baseline & After change in arterial pressure & Postinfusion & $\mathrm{P}$ value $^{\mathrm{a}}$ \\
\hline \multicolumn{5}{|l|}{$\mathrm{EA}_{\mathrm{dyn}}$} \\
\hline HighMAP & $0.92 \pm 0.12$ & $0.52 \pm 0.23^{*}$ & $0.49 \pm .0 .18$ & $<0.001$ \\
\hline LOWMAP & $0.91 \pm 0.16$ & $1.64 \pm 0.44^{*}$ & $1.26 \pm 0.22^{\dagger *}$ & \\
\hline \multicolumn{5}{|c|}{$\mathrm{Ea}, \mathrm{cmHg} / \mathrm{mL}$} \\
\hline HighMAP & $3.99 \pm 0.93$ & $5.42 \pm 1.46^{*}$ & $4.45 \pm 1.11^{\dagger}$ & $<0.001$ \\
\hline LOWMAP & $4.50 \pm 1.44$ & $1.80 \pm 0.63^{*}$ & $1.52 \pm 0.50^{+^{*}}$ & \\
\hline \multicolumn{5}{|c|}{$\mathrm{C}, \mathrm{mL} / \mathrm{cmHg}$} \\
\hline HighMAP & $0.66 \pm 0.13$ & $0.41 \pm 0.10^{*}$ & $0.48 \pm 0.08^{\dagger^{*}}$ & $<0.001$ \\
\hline LOWMAP & $0.53 \pm 0.16$ & $1.92 \pm 0.86^{*}$ & $1.83 \pm 0.58^{*}$ & \\
\hline \multicolumn{5}{|c|}{ TVSR, MPa's $/ \mathrm{m}^{3}$} \\
\hline HighMAP & $1514 \pm 453$ & $3157 \pm 1299^{*}$ & $3372 \pm 1306^{*}$ & $<0.001$ \\
\hline LOWMAP & $1814 \pm 585$ & $709 \pm 215^{*}$ & $720 \pm 218^{*}$ & \\
\hline
\end{tabular}

C: new arterial compliance; Ea: effective arterial elastance; Eadyn: dynamic arterial elastance; TSVR: total systemic vascular resistance. Data are presented as mean \pm standard deviation. Data normally distributed according to Kolomogorov-Smirnov test. Note that pressure units are expressed as cmHg for convenience.

${ }^{*} p<0.05$ vs baseline. ${ }^{\dagger} p<0.05$ vs. change in arterial pressure. ${ }^{*} p$ value refers to ANOVA test for time and group interaction

\section{Methods}

12 anesthetized and mechanically ventilated rabbits. Arterial tone changes were induced by phenylephrine (PHENY) infusion on 6 animals (HighMAP group) and by sodium nitroprusside (SNP) on the other 6 animals (LowMAP group), until reach a $50 \%$ of change on mean arterial pressure (MAP) from its baseline value. A volume challenge $(10 \mathrm{~mL} / \mathrm{Kg})$ was then performed on all animals. Animals were monitored with an indwelling femoral arterial catheter and an esophageal Doppler (CardioQCombi). Arterial load was assess by the systemic vascular resistance, net arterial compliance and effective arterial elastance. $\mathrm{Ea}_{\mathrm{dyn}}$ was calculated as the simultaneous ratio between PPV and SVV obtained from the Doppler monitor.

\section{Results}

At baseline, $\mathrm{Ea}_{\mathrm{dyn}}$ and other arterial load parameters were similar on both groups. In the LowMAP group, SNP significantly decreased arterial load, reduced MAP by $44 \%$, and consistently increased Eadyn by $75 \%$ (Figure 1). In the HighMAP group, PHENY increased arterial load, raised MAP by $58 \%$, and significantly reduced $\mathrm{Ea}_{\mathrm{dyn}}$ by 41\% (Fig. 1 and 2). Overall, VE increased cardiac output by $10 \%$, stroke volume by $21 \%$ and MAP by $15 \%$, and decreased $\mathrm{Ea}_{\mathrm{dyn}}$ from $1.08 \pm 0.67$ to $0.88 \pm 0.45$ (Fig.1). 
There was a significant relationship between $\mathrm{Ea}_{\mathrm{dyn}}$ after arterial tone changes and increases in all components of arterial pressure after VE: systolic $\left(\mathrm{R}^{2}=0.89\right)$, diastolic $\left(R^{2}=0.41\right)$, mean arterial $\left(R^{2}=0.61\right)$ and pulse pressure $\left(R^{2}=0.67\right)$, respectively. Animals with a MAP increase $\geq$ $10 \%$ after VE had a higher preinfusion $\mathrm{Ea}_{\mathrm{dyn}}$ value (1.54 \pm 0.49 vs. $0.46 \pm 0.15 ; \mathrm{P}<0.001)$.

\section{Conclusions}

In this experimental settings acute modifications on arterial tone induced significant changes on $\mathrm{Ea}_{\mathrm{dyn}}$ : arterial vasodilation increased $\mathrm{Ea}_{\mathrm{dyn}}$, whereas vasoconstriction decreased it. Nevertheless, preinfusion $\mathrm{Ea}_{\text {dyn }}$ still determined the arterial pressure response after volume administration.

\section{Authors' details}

'St. George's Healthcare NHS Trust and St George's University of London, Department of Intensive Care Medicine, London, UK. ${ }^{2}$ Hospital SAS de Jerez, Servicio de Cuidados Intensivos y Urgencias, Jerez de la Frontera, Spain.

${ }^{3}$ Universitat Autónoma de Barcelona, Consorci Sanitari Universitari Parc Tauli, Barcelona, Spain.

Published: 26 September 2014

\section{References}

1. Monge Garcia MI, Gil Cano A, Gracia Romero M: Dynamic arterial elastance to predict arterial pressure response to volume loading in preloaddependent patients. Crit Care 2011, 15:R15.

2. Hadian M, Severyn DA, Pinsky MR: The effects of vasoactive drugs on pulse pressure and stroke volume variation in postoperative ventilated patients. Journal of critical care 2011, 26(328):e321-328.

doi:10.1186/2197-425X-2-S1-P35

Cite this article as: García et al:: 0590. Impact of arterial tone changes on dynamic arterial elastance and the arterial pressure response to fluid administration. Intensive Care Medicine Experimental 2014 2(Suppl 1):P35.

\section{Submit your manuscript to a SpringerOpen ${ }^{\circ}$ journal and benefit from:}

- Convenient online submission

- Rigorous peer review

- Immediate publication on acceptance

- Open access: articles freely available online

- High visibility within the field

- Retaining the copyright to your article

Submit your next manuscript at $\gg$ springeropen.com 